\title{
Whole-Body Vibration Improves the Accuracy of Motor Performance
}

\section{Nina Völkel* and Ewald M. Hennig}

Biomechanics Laboratory, University Duisburg-Essen, Gladbecker Str. 182, 45141 Essen, Germany

\begin{abstract}
Several studies showed changes in motor control performance following whole-body vibrations. It is difficult to compare the results and conclusions from studies in the literature, because they often use different experimental protocols. We conducted a series of four studies with a similar protocol to investigate the acute effect of whole-body vibrations on balance control, accuracy of movement execution, and cognitive attention and concentration. We found an increase in balancing time for a one-leg standing task with closed eyes. Two subsequent studies revealed a better accuracy in motor performance for jumping onto a target and improved hand coordination during the task of aiming with a laser pointer onto a target. In the fourth study no acute changes in cognitive attention and concentration was found following whole-body vibrations. Therefore, it appears that motor control areas in the brain benefit from whole-body vibrations whereas more complex brain structures do not cause an increase in attention or cognitive performance.
\end{abstract}

Keywords: Whole-body vibration, Balance control, Movement accuracy, Hand coordination, Motor performance, Cognitive attention, Concentration

\section{Introduction}

Whole-body vibration (WBV) training becomes increasingly popular in fitness studios and rehabilitation centres. It can serve to augment the performance and training of athletes [1]. The human body reaction, based on a WBV treatment, depends on the character and frequency of the WBV [2]. Muscle spindles can be excited by WBV stimuli and cause muscle contraction [2]. The reaction of the human body to a WBV stimulus is multifaceted and non-linear with the excitation amplitude [3]. The absorption of the WBV stimulus in the human body is one reason for this effect. This absorption also depends on foot positioning on the platform [3].

During whole-body vibration stochastic resonance phenomena are believed to occur [4]. In the literature this phenomenon is described as a short-term interference mechanism between the natural stochastic part of the neuron and an external stochastic part [5]. A modification in the neurotransmitter concentration in the brain has also been speculated to occur with WBV [6].

Motivated by the findings from Parkinson studies on the effect of WBV on motor performance [6] we wanted to find out, whether an improvement in motor performance can also be observed in non pathological populations. If this is the case, athletes and the general population may benefit from WBV training.

We conducted a series of four studies to analyse the acute effects of a whole-body vibration treatment on balance control, accuracy of landing on a target, precision of hand coordination, and the effect on cognitive brain performance. The experimental procedures in these studies were kept similar to provide a base for a comprehensive and comparative discussion of our results.

\section{Methods}

\section{Subjects}

All of our studies were conducted with healthy sport student volunteers from the University Duisburg-Essen. Each volunteer was given written summary and oral information about the testing procedures. The participants were required to sign the consent form indicating voluntary participation in accordance with University of Duisburg-Essen policy.

\section{Whole-body-vibration (WBV)}

For the treatment we used whole-body vibration platforms based on the principle of a side alternating motion and generating a sinusoidal stimulus. In two studies we used the "Galileo Fitness" platform by Novotec Medical (Pforzheim, Germany) and for the other two studies the "Qionic-Board Classic" by QIONIC GmbH (Burtenbach, Germany).

The treatment consisted of a 2 minute WBV training with two different frequencies. The low frequency was $5 \mathrm{~Hz}$ in all studies. The high frequency was $26 \mathrm{~Hz}$ for the studies with the Galileo platform and $25 \mathrm{~Hz}$ for the studies with the Qionic device.

During the first minute the subjects stood freely on the platform and performed dynamic knee bends without shoes. The amplitude of the WBV depends on foot position on the platform. The further the feet stand apart from each other, the higher is the amplitude. The subjects stood always on foot position 2, as marked on the vibration standing plate. This foot position translates on the Galileo platform to a peakto-peak displacement of $5.4 \mathrm{~mm}$ and on the Qionic Board to $4 \mathrm{~mm}$ for both frequencies. The peak acceleration values were for the Galileo platform $\mathrm{a}_{\text {peak, }} 5 \mathrm{~Hz}=0.27 \mathrm{~g}, \mathrm{a}_{\text {peak, }}, 26 \mathrm{~Hz}=6,79 \mathrm{~g}$ and for the Qionic Board $\mathrm{a}_{\text {peak, }}, 5 \mathrm{~Hz}=0.20 \mathrm{~g}$, a peak $^{\text {peak }}, 26 \mathrm{~Hz}=5,03 \mathrm{~g}$. A test of "Skidding" [7] showed no skidding effect for both vibration frequencies on the two platforms. For the second minute of WBV application the subjects sat on the platforms.

\section{Statistical Methods}

For statistical evaluation we used analysis of variance (ANOVA) methods with post-hoc t-tests.

\section{Balance}

In our first study [8] we tested the influence of a WBV treatment on a static balance task with 19 female and 24 male young and healthy

*Corresponding author: Nina Völkel, Biomechanics Laboratory, University Duisburg-Essen, Gladbecker Str. 182, 45141 Essen, Germany, Tel: +49-201-1837350; Fax: +49-201-183-7309; E-mail: nina.voelkel@uni-due.de

Received May 16, 2012; Accepted June 22, 2012; Published June 25, 2012

Citation: Völkel N, Hennig EM (2012) Whole-Body Vibration Improves the Accuracy of Motor Performance. J Sport Medic Doping Studie S7:001. doi:10.4172/21610673.S7-001

Copyright: (c) 2012 Nina V, et al. This is an open-access article distributed under the terms of the Creative Commons Attribution License, which permits unrestricted use, distribution, and reproduction in any medium, provided the original author and source are credited. 
Citation: Völkel N, Hennig EM (2012) Whole-Body Vibration Improves the Accuracy of Motor Performance. J Sport Medic Doping Studie S7:001. doi:10.4172/2161-0673.S7-001

sport students from the University Duisburg-Essen. Their mean age was $23.4 \pm 3.0$ years.

In the literature it is reported that a WBV treatment with $6 \mathrm{~Hz}$ improves balance performance in Parkinson patients [9]. We wanted to investigate whether balance control improvement after WBV can also be observed in healthy sport students.

The subjects came on two different days and were instructed to stand as long as possible on one leg with closed eyes on an "Airex Balance Pad" with a thickness of $6 \mathrm{~cm}$. Balancing duration was determined as follows. Time was measured from the moment the subjects closed their eyes on the balance pad until they lost balance by putting down their second leg for balance recovery. The time was measured with an electronic stopwatch.

Prior to the WBV treatment, the subjects repeated the balance task 7 times (Pre measurement). Thereafter, a WBV treatment followed on the "Galileo" platform. Immediately after the WBV the second balance measurement (Post) followed. The whole-body vibration treatment of 5 $\mathrm{Hz}$ and $26 \mathrm{~Hz}$ was randomized between subjects on both test days. The mean values from the 7 Pre and 7 Post balance task were calculated and used for statistical evaluation.

Results: The statistical analysis did not show any gender differences in the results for either the Pre and Post measurements or the treatment effects. Therefore, the following results are based on the statistical analysis of the 43 male and female sport students.

Statistically significant improvements $(\mathrm{p}<0.01)$ in balancing time were found after both the $5 \mathrm{~Hz}$ and $26 \mathrm{~Hz}$ WBV treatments. There were no significant differences between the two treatment conditions. The values in figure 1 are normalized to the Pre condition in percent. The mean standing time in the Pre condition for all subjects was $8.15 \mathrm{~s}$.

Our first study showed that whole-body vibrations with $5 \mathrm{~Hz}$ and $26 \mathrm{~Hz}$ have an acute effect on balance control during a standing task in healthy sport students.

To maintain static balance the body needs information from kinesthetic, tactile and optical analysers. In our task information from the optical analyser was removed, because the subjects closed their eyes. For Parkinson patients a WBV treatment improved motor coordination performance [6]. The study explored ad-hoc reactions following WBV stimuli with 4 to $6 \mathrm{~Hz}$. They found a reduction in gait impairment. Motivated by these results we used for our second study a more complex

\section{Balancing time improvement after WBV}

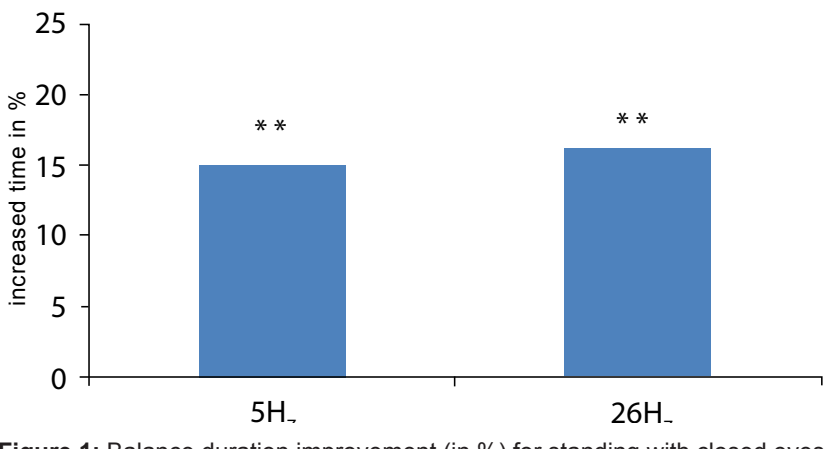

Figure 1: Balance duration improvement (in \%) for standing with closed eyes on one leg before (Pre) and after (Post) WBV treatments with $5 \mathrm{~Hz}$ and 26 $\mathrm{Hz}\left({ }^{* *} \mathrm{p}<0.01\right)$

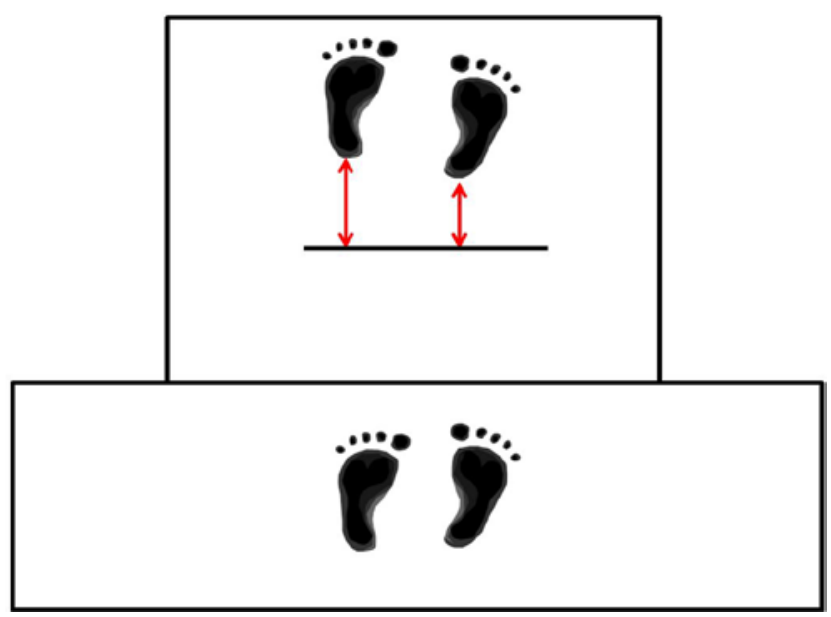

Figure 2: Experimental set-up for jumping accuracy after WBV.

and dynamic motor task to explore whether WBV also has a positive effect on the motor performance of healthy sport students.

\section{Accuracy in a jumping task}

20 female and 20 male sport students from the University DuisburgEssen participated in this study [10]. Their mean age was $24.5 \pm 2.2$ years.

The chosen movement task was adapted from a children's coordination test (KiKo) [11]. Jumps for best landing accuracy were performed from a table (height $=70 \mathrm{~cm}$ ) onto a target, placed on a firm landing mat. A target line in jumping direction was painted on the mat. The distance between table and the target line was chosen differently for women and men. In a pilot study jumps for maximum distance were performed with women and men. Using the means of these distances for both genders, the target line was set at $60 \%$ of these values. From this procedure the distance for the female subjects was set at $120 \mathrm{~cm}$ and for the male subjects at $155 \mathrm{~cm}$.

All subjects were instructed to jump from the table and land as accurately as possible with the back of their heels on the target line. The subjects were free in choosing their preferred standing position on the table. However, they had to jump off and land with both feet. After landing the subjects were instructed to stand still until the measurements with a ruler were finished. The absolute distances from both heels to the target line were measured and averaged (Figure 2).

In addition to the two different WBV treatments on the "Galileo Fitness" platform $(5 \mathrm{~Hz}, 26 \mathrm{~Hz})$ a third treatment was introduced for this study. This task had also duration of 2 minutes, and the subjects were asked to detect figures in complex 3-D patterns. We told the subjects, that this task could serve to improve their concentration and may be beneficial for jumping more accurately.

Five jumps were performed before the treatment (Pre) in each of three test days. Thereafter, a treatment $(5 \mathrm{~Hz}, 26 \mathrm{~Hz}$ or $3-\mathrm{D}$ patterns) and a second set of 5 jumps (Post) followed. The treatment was randomized for the subjects across the three days. The mean values of the absolute target deviation values from the 5 Pre and 5 Post jumps were calculated for each individual and used for further statistical evaluation.

Results: A statistically significant improvement $(p<0.05)$ in landing precision was found after the $5 \mathrm{~Hz}$ whole-body vibration treatment. No significant changes were observed after the $26 \mathrm{~Hz}(\mathrm{p}=$ 
Citation: Völkel N, Hennig EM (2012) Whole-Body Vibration Improves the Accuracy of Motor Performance. J Sport Medic Doping Studie S7:001. doi:10.4172/2161-0673.S7-001

Page 3 of 5

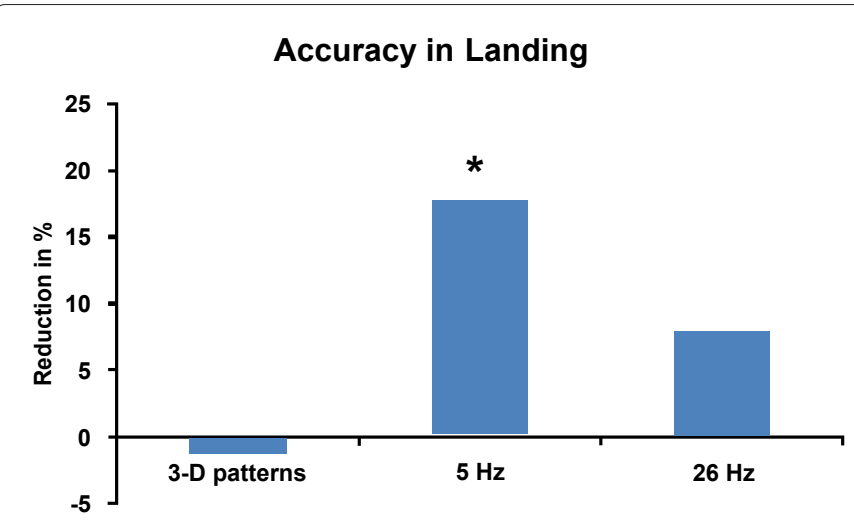

Figure 3: Percent difference in the deviation from precision jumps onto a target before (Pre) and after (Post) WBV treatments with $5 \mathrm{~Hz}$ and $26 \mathrm{~Hz}\left({ }^{*} \mathrm{p}\right.$ $<0.05)$.

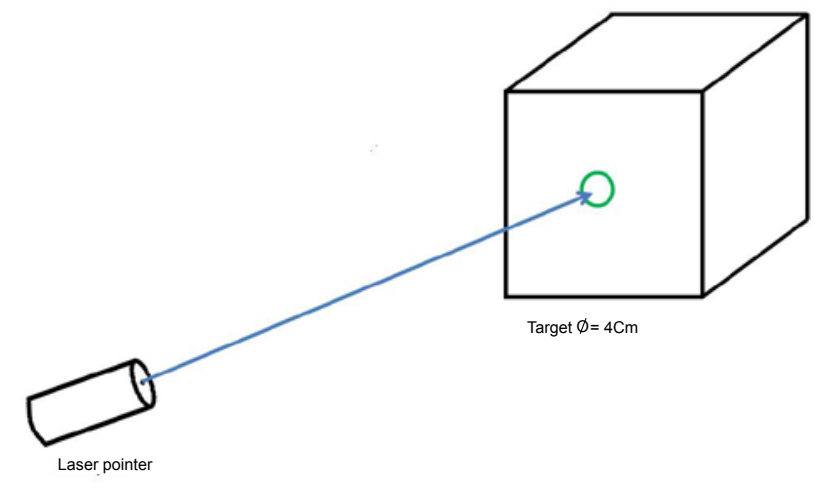

Figure 4: Experimental set-up for hand coordnation measurements.

$0.254)$ and the placebo condition. The values in figure 3 are normalized to the Pre condition in percent. The absolute distance from the target line across all subjects and the three jumping conditions was $4.00 \mathrm{~cm}$.

Only the lower frequency showed significant changes in the accuracy of landing, although there is also a trend for better precision after the $26 \mathrm{~Hz}$ WBV treatment. The low frequency results are in line with the findings for Parkinson patients [6]. The study also showed an improvement of handwriting skills for their Parkinson patients [6]. Tremor was reduced and the handwriting was much clearer. With our third study we wanted to find out whether improved hand coordination can also be observed in healthy persons.

\section{Hand coordination}

20 female and 20 male sport students from the University DuisburgEssen participated in this study [12]. Their mean age was $24.2 \pm 3.4$ years.

All subjects sat on a chair with a laser pointer in their hand. At a distance of $10 \mathrm{~m}$ a $1 \mathrm{~m} \times 1 \mathrm{~m} \times 1 \mathrm{~m}$ box was positioned. The front plane of the box was made of Plexiglas with a target in its centre. The subjects were instructed to point with a laser pointer to the target as accurate and steady as possible for a duration of $20 \mathrm{~s}$ (figure 4).

The position and trace of the laser beam on the Plexiglas surface was recorded with a video camera at a frequency of $300 \mathrm{~Hz}$ from behind. We measured the length of the laser beam trace and its absolute deviation from the target centre for the middle 15 second period within the 20 second video recording.

The subjects performed the tests on two different days. After a 5 minute warm-up on a bicycle ergometer the subjects performed 3 times the measurement (Pre). The WBV treatment with the "Qionic Board" followed. Thereafter, we carried out a second measurement of the hand movement (Post). The assignment of the treatments (5 Hz, 25 $\mathrm{Hz}$ ) between the two days was randomized for the subjects. The mean values from the 3 Pre and 3 Post measurements were calculated and used for statistical evaluation.

Results: When comparing the Pre conditions between the two different days there were no statistically significant differences for both the trace length of the laser beam and the absolute deviation from the target centre. Therefore, no learning effect was present from the first to the second day.

After the WBV with $5 \mathrm{~Hz}$ a statistically significant reduction of the trace length of the laser beam $(\mathrm{p}<0.01)$ was observed (Figure 5). Similarly, the absolute deviation of the laser beam towards the target centre was significantly reduced after the $5 \mathrm{~Hz}$ WBV treatment ( $\mathrm{p}<$ 0.01) (Figure 6). No significant changes were found after the $25 \mathrm{~Hz}$ treatment.

Across all subjects and the two treatments the average trace length of the laser beam during one second was $21.92 \mathrm{~cm}$. From 300 frames of

\section{Trace Length of the Laser Beam}

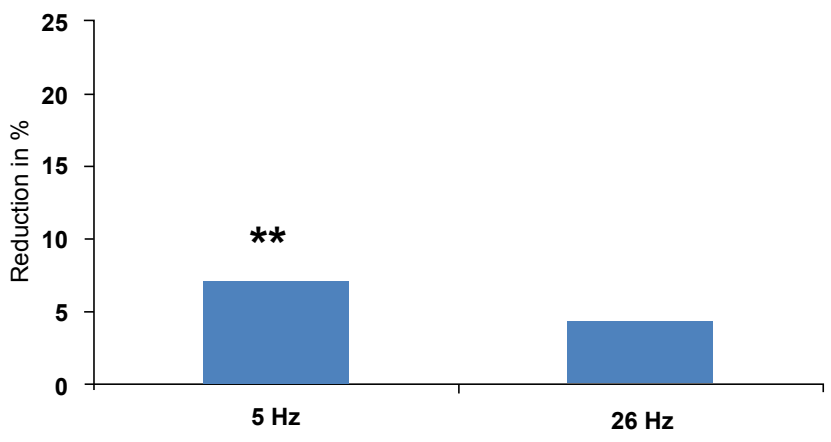

Figure 5: Percent difference in the trace length before (Pre) and after (Post) WBV treatments with $5 \mathrm{~Hz}$ and $26 \mathrm{~Hz}\left({ }^{* *} \mathrm{p}<0.01\right)$

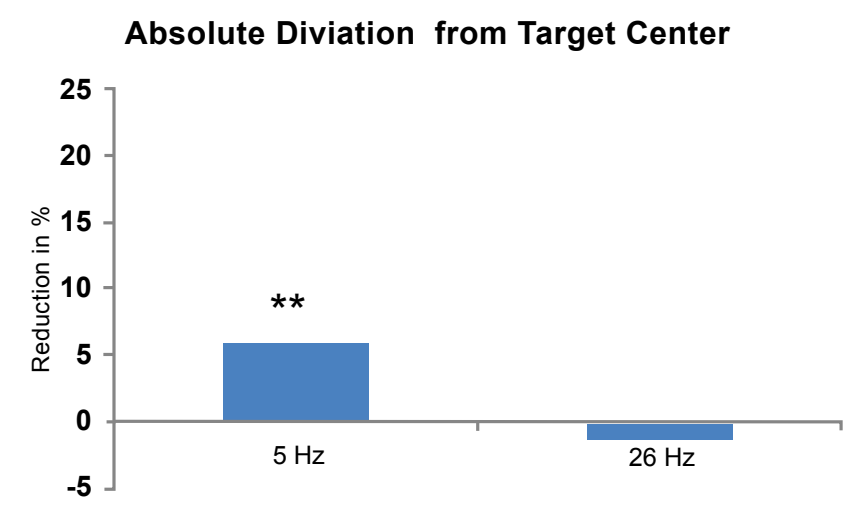

Figure 6: Percent difference in absolute deviation from the target center before (Pre) and after (Post) WBV treatments with $5 \mathrm{~Hz}$ and $26 \mathrm{~Hz}$ (** $\mathrm{p}<$ 0.01). 
1 second video recording the sum of the absolute distance deviations from the target centre was $53.76 \mathrm{~cm}$.

The above results fit well with the findings of improved hand writing abilities in Parkinson patients [6]. The authors reported improvements in hand coordination of their patients after low frequency WBV [6]. The results from our first three studies suggest that higher central nervous structures are involved in the improvements of balance and precision of movement execution after WBV. Stochastic resonance phenomena $[4,5]$ and a modification of neurotransmitter concentration [6] in the brain have been suggested as explanations for the improvements in motor abilities after WBV. From these hypotheses the question arises whether these changes in brain function are restricted to motor performance. Therefore, in our fourth study, we wanted to explore whether WBV also has an effect on cognitive attention and concentration.

\section{Attention and concentration}

60 male sport students from the University Duisburg-Essen participated in our fourth study [13]. Their mean age was $25.0 \pm 2.7$ years. They were randomly assigned to an experimental and a control group. The 30 subjects of each group started with a 5 minute warmup on a bicycle ergometer followed by Pre attention and concentration measurement. Thereafter they performed three psychological personality trait tests. The tests were about intelligence valuator, neuroticism and a test about cognitive estimation. After these tests the subjects carried out a simple manual task. This task was introduced to guarantee a constant break time of 35 minutes for all subjects between the Pre and Post measurements. After this break a second 5 minute warm-up and the treatment followed. Both groups performed their exercises on the Qionic-Classic Board. The experimental group with a frequency of $5 \mathrm{~Hz}$ and the control group with $0 \mathrm{~Hz}$. Thereafter, the Post attention and concentration measurements followed. Attention and concentration were tested with the D2, Stroop and Trail Making Test. The D2 [14] is an attention test, and subjects are required to recognize and mark certain objects during a given time period. We measured the number of mistakes in percent (accuracy) and the number of worked items (speed). The third parameter (concentration) can be calculated from accuracy and speed [14].

The Stroop test $[15,16]$ is a colour word interference test and consists of three subtests from which we only used the last one. In this test coloured words do not coincide with the meaning of the word (e.g. the word red is coloured green). The subjects had to name the colour of the written word to the test administrator. The required time for naming a list of words and the number of mistakes were recorded $[15,16]$.

The Trail Making Test [17] consisted of two tests. In the first test, the subjects had to connect a set of 25 dots as fast and accurate as possible with a pencil. In the second test, they had to connect numbers with letters. For both tests performance times were evaluated.

Results: Between the two groups the psychological tests did not show a difference in personal trait between our experimental and control group. For all attention and concentration tests an improvement between Pre and Post measurements was present for the experimental as well as the control group. In the D2 test the subjects worked more items and made less mistakes. The times for the Stroop test and the Trail Making Test were reduced. However, there were no statistically significant differences between the control and experimental group for all attention and concentration tests. These results indicate that a learning effect was present for both groups. However, in comparison to the control group the $5 \mathrm{~Hz}$ WBV of our experimental group did not result in a better performance.

\section{Discussion and Conclusions}

The changes in balance performance agree with results from other authors [18]. These authors found that a 4 minute WBV at $15-30 \mathrm{~Hz}$ improves the balance of young and healthy persons.

The improvement in the accuracy of landing and hand coordination after the $5 \mathrm{~Hz}$ treatment is likely to be caused by a similar mechanism that improved gait and handwriting for Parkinson patients after WBV [6]. The authors also mention increases of neurotransmitter concentration in the brain as an additional explanation [6]. Animal experiments did show that changes in neurotransmitter concentration in the brain occurred after vibration stimuli. The concentration in noradrenalin increases after the vibration treatment in the brain of rats [19].

Another explanation for the performance enhancement after low frequency treatment can be short-term neural mechanisms. The more accurate movement after the $5 \mathrm{~Hz}$ treatment may be explained by the introduction of noise, causing stochastic resonance effects in the central nervous system [20]. Other authors described this phenomenon with interference between the natural stochastic parts of the neurons and the external vibration stimulus [5].

The results of our studies show, that WBV training on a "Galileo Fitness" and "Qionic-Classic Board" platform enhances the precision of landing on a target and improves hand coordination. However, this improvement is only statistically significant after a low frequency WBV at $5 \mathrm{~Hz}$.

The whole-body vibration did not result in an improvement of cognitive attention and concentration, as measured with the D2, Stroop and Trail Making Tests.

This suggests that whole-body vibrations have an effect on the function of motor performance related brain structures. Effects on higher level cognitive and attention related brain performance could not be detected by our psychological measurement tests.

A limitation of our studies is the restriction to acute effects of WBVs on motor performance. Furthermore, our studies were performed with physically active and young sport students. Whether our findings will also apply to a general population remains an open question.

Improving accuracy and balance control has manifold and obvious applications for performance improvement in many sports. An improvement in motor performance has been reported for Parkinson patients [6] and was also found in our studies. There seems to be a general benefit in motor performance related brain function, which is not only limited to a pathological population. Therefore, low frequency whole-body vibrations could also be a training modality for the prevention of falls in the elderly. This field of application should be explored in more detail, because it would have an important impact on health care and life quality.

Future research should explore long term effects of WBV on motor performance. Can a regularly performed WBV training produce long-term effects of precision improvement? For Parkinson patients a positive effect of WBV lasted from several hours up to two days in some subjects [6].

\section{References}

1. Jordan MJ, Norris SR, Smith DJ, Herzog W (2005) Vibration training: an overview of the area, training consequences, and future considerations. J Strength Cond Res 19: 459-466. 
Citation: Völkel N, Hennig EM (2012) Whole-Body Vibration Improves the Accuracy of Motor Performance. J Sport Medic Doping Studie S7:001. doi:10.4172/2161-0673.S7-001

2. Burkhardt A (2006) Vibrationstraining in der Physiotherapie. Physiopraxis 9: $22-25$

3. Haas CT, Turbanski S, Kaiser I, Schmidtbleicher D (2004) Biomechanische und physiologische Effekte mechanischer Schwingungsreize beim Menschen. Deutsche Zeitschrift für Sportmedizin 55: 34-43.

4. Moos F, Ward LM, Sannita WG (2004) Stochastic resonance and sensory information processing: a tutorial and review of application. Clin Neurophysiol 115: $267-281$.

5. Haas CT (2008) Vibrationstraining, Biomechanische Stimulation und Stochastische Resonanz Therapie. Zeitschrift für Physiotherapeuten 60: 728740.

6. Haas CT, Schmidtbleicher D (2002) Zu den Effekten mechanischer Schwingungsreize bei Morbus Parkinson. Rheuma Aktuell 3: 8-10.

7. Rauch F, Sievanen H, Boonen S, Cardinale M, Degens H, et al. (2010) Reporting whole-body vibration intervention studies: Recommendations of the International Society of Musculoskeletal and Neuronal Interactions. J Musculoskelet Neuronal Interact 10: 193-198.

8. Hennig E, Willems H (2011) Der Akuteinfluss von Ganzkörpervibrationen auf das Gleichgewichtsverhalten beim Einbeinstand. In Siebert T, Blickhan $\mathrm{R}$ (Hrsg.) Biomechanik - vom Muskelmodell bis zur angewandten Bewegungswissenschaft. Jena: Friedrich-Schiller-Universität Jena, p. 21.

9. Turbanski S, Haas CT, Schmidtbleicher D, Friedrich A, Duisberg P (2005) Effects of whol-body vibration on postural control in Parkinson's disease. Res Sports Med 13: 243-256.

10. Völkel N, Hennig EM (2010) Acute Effects of Whole Body Vibration on Accuracy of Motor Performance. In Stevenson J (Ed.) 16th Biannual Conference of the Canadian Society of Biomechanics. Kingston, Ontario, Canada: Queens University, p. 52.

11. Prätorius B (2008) Entwicklung eines Koordinationstests für Kinder im
Grundschulalter und dessen Validierung mit Hilfe biomechanischer Methoden. Dissertation Technische Universität Chemnitz, Institut für Sportwissenschaften Chemnitz.

12. Völkel N, Froeschke C, Hennig E (2011) Akuteffekt eines Vibrationstrainings auf die Handkoordination. In Hottenrott K, Stoll O, Wollny R (Hrsg.) Schriften der Deutschen Vereinigung für Sportwissenschaft Band 215: Czwalina Verlag Hamburg, p. 257.

13. Völkel N, Letailleur J, Hennig E, Schulte F (2012) Beinflusst ein Vibrationstraining die Aufmerksamkeit? In Wagner H. (Hrsg.) NeuroMotion - Aufmerksamkeit, Automatieiserung, Adaptation. Münster: Westfälische Wilhelms-Universität Münster, p. 53

14. Brickenkamp R, Schmidt-Atzert L, Liepmann D (1994) Test d2. Hogrefe.

15. Bäumler G (1984) Farbe-Wort-Interferenztest (FWIT) nach J.R. Stroop, Hogrefe.

16. Amelang M, Schmidt-Atzert L (2006) Psychologische Diagnostik und Intervention Springer, Verlag.

17. Bowie CR, Harvey P (2006) Administration and interpretation of the Trail Making Test. Nat Protoc 1: 2277-2281.

18. Torvinen S, Kannus P, Sievänen H, Järvinen TAH, Pasanen M, et al. (2002) Effect of a vibration exposure on muscular performance and body balance. Randomized cross-over study. Clin Physiol Funct Imaging 221: 145-152.

19. Ariizumi M, Okada A (1985) Effects of whole body vibration on biogenic amines in rat brain. $\mathrm{Br} \mathrm{J}$ Ind Med 42: 133-136.

20. Hänggi P (2001) Stochastische Resonanz: Rauschen macht sensibel. Physikalische Blätter 57: 15-16.

This article was originally published in a special issue, Biomechanics handled by Editor(s). Dr. Damiano Marchi, University of the Witwatersrand, South Africa 